\title{
De la Web social al Móvil 2.0: el paradigma 2.0 en el proceso de convergencia mediática de la comunicación móvil
}

\author{
Por Juan-Miguel Aguado-Terrón e Inmaculada J. Martínez-Martínez
}

\begin{abstract}
Resumen: En virtud de su capacidad creciente de conectividad, procesamiento y transmisión de datos, el dispositivo móvil se encuentra inmerso en un proceso de convergencia con el ecosistema mediático, que involucra, por un lado, a paradigmas propios de los medios convencionales (contenidos estandarizados, coste por acceso, consumo individualizado pasivo) y por otro, al paradigma característico de la Web Social (contenidos personalizados y/o generados por el usuario, entornos abiertos a terceras partes, trabajo colaborativo, predominio de la cultura de la compartición, etc.). El presente artículo propone un marco general de los factores relevantes por los que el paradigma de la Web Social se perfila actualmente como un modelo prevalente en el desarrollo del mercado y el consumo de datos en dispositivos móviles, aportando una estructura conceptual de utilidad para el posterior estudio evolutivo de los modelos de producción, negocio y consumo de los contenidos móviles.
\end{abstract}

Palabras clave: Dispositivo móvil, Paradigma 2.0, Web móvil, Conectividad ubicua, Adaptación al contexto, Geolocalización, Redes sociales, Mobile 2.0.

Title: From Social Web to Mobile 2.0: The 2.0 paradigm in the mediatization process of mobile communications

Abstract: Due to their increasing connectivity and processing power, mobile devices are engaged in a process of mediatization that involves, on one side, conventional media related paradigms (standardized contents, pay per access, individualized passive consumption) and, on the other side, the Social Web related 2.0 paradigm (personalized and user generated content, third party open environments, collaborative work, prevalence of 'sharing' logics, etc.). This paper poses a general framework of those factors that enable the relevance of the Web 2.0 paradigm in the current development of market and data consumption in mobile devices, including some conceptual outline that may prove useful in future research of the evolution of mobile content production, business and consumption models.

Keywords: Mobile devices, 2.0 paradigm, Mobile Web, Ubiquitous connectivity, Context sensitivity, Geo-location, Social networks, Mobile 2.0.

Aguado-Terrón, Juan-Miguel; Martínez-Martínez, Inmaculada J. “De la Web social al Móvil 2.0: el paradigma 2.0 en el proceso de convergencia mediática de la comunicación móvil". El profesional de la información, 2009, marzo-abril, v. 18 , n. 2 , pp. $155-161$.

DOI: 10.3145/epi.2009.mar.05

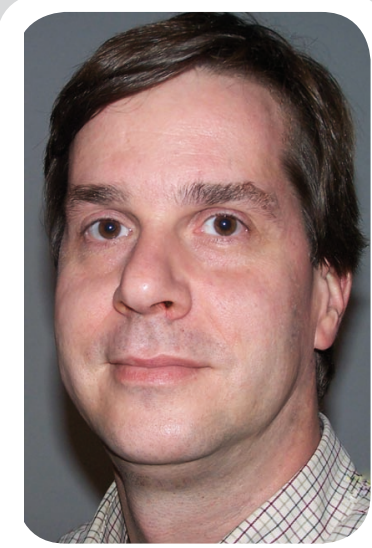

Juan Miguel Aguado, doctor en ciencias de la información por la Universidad Complutense de Madrid y posgrado en investigación social por la Polish Academy of Sciences (Varsovia), es profesor titular de teoría de la comunicación en la Facultad de Comunicación y Documentación de la Universidad de Murcia. Actualmente dirige el proyecto de investigación Movilsoc "El impacto social de la telefonía móvil en España: gestión de identidad, mediatización y ritos de consumo" en el marco del Grupo de Investigación en Comunicación Social, Cultura y Tecnología (E-COM), del que es fundador.

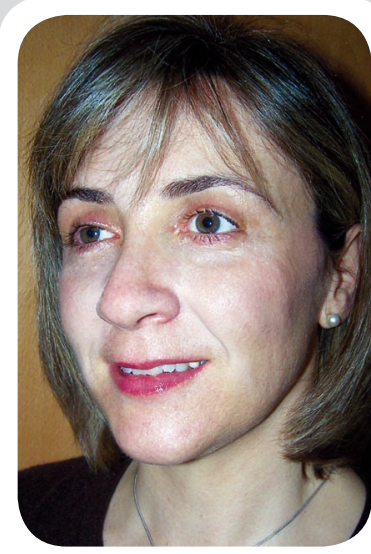

Inmaculada J. Martínez, formación por la Universidad Complutense de Madrid y master en dirección y gestión de empresas por la Know How Business School, ha realizado una extensa labor de consultoría y formación "in company" sobre estrategias de imagen y comunicación en España, Brasil y Portugal. Es miembro fundador del E-COM, en el que participa en el proyecto Movilsoc y dirige un proyecto $I+D$ sobre mujer y tecnologías móviles. Es profesora titular de publicidad y relaciones públicas en la Facultad de Comunicación y Documentación de la Universidad de Murcia. doctora en ciencias de la in-

\section{Introducción}

En virtud de su creciente capacidad de conectividad, procesamiento y transmisión de datos, el dispositivo móvil (teléfonos, smartphones, PDAs...) deviene un medio de consumo que a su condición de meta-dispo- sitivo tecnológico (cámara, agenda, TV, videoconsola, ordenador, reproductor de audio/vídeo, GPS...) suma los rasgos de un objeto cultural fuertemente ligado a la identidad del usuario y un medio para la producción, distribución y consumo de datos y contenidos (Katz; Satomi, 2005; Fortunati, 2006; Aguado; Martínez, 
2006; 2007). Precisamente por su ubicuidad y por su vinculación a la identidad, el móvil ofrece una gran capacidad de adaptación al usuario y al contexto de uso (pertinencia/conveniencia). Estos rasgos constituyen, de hecho, el principal atractivo para la implicación de las industrias de la información en el desarrollo de las comunicaciones móviles (Aguado; Martínez, 2007). A partir de ellos, la plataforma móvil se perfila como una suerte de tierra prometida informacional en la que el acceso a datos carecería de limitaciones contextuales: 'lo que quieras, cuando quieras, donde quieras'.

Ese interés creciente responde además a tres factores específicos que determinan tanto sus horizontes como sus límites:

- la saturación del mercado en el ámbito de la comunicación por voz y la creciente importancia de los servicios de datos (Telefónica, 2008; CMT, 2007; Spurgeon; Goggin, 2006);

- la convergencia en el dispositivo móvil de la doble condición de plataforma de comunicación -marcada por la confluencia con los estándares de internet y por el desarrollo de la multi-conectividad con otros dispositivos (UMTS, USB-PC, wifi, Bluetooth, infrarrojos, etc.)- y plataforma de contenidos -marcada por su creciente versatilidad multimedia- (Aguado; Martínez, 2008);

- dada su vinculación identitaria con el usuario, el interés estratégico de las comunicaciones móviles como valor añadido para la imagen de marca de los actores del sector mediático y como vínculo de conexión entre diversos formatos y rutinas de consumo de contenidos (Feldmann, 2005).

El resultado es la gestación de un variado paisaje de servicios y productos vinculados a la gestión de información móvil (entretenimiento, gestión y edición de contenidos, información sensible a la localización y al contexto, publicidad y marketing móvil, etc.), que a su vez constituye el aspecto más visible de un creciente proceso de mediatización -entendido como convergencia con los formatos y modelos del ecosistema mediático- del dispositivo móvil (Aguado; Martínez, 2007). Este proceso involucra, por un lado, a paradigmas propios de los medios tradicionales (contenidos estandarizados, coste por acceso, consumo individualizado pasivo) y por otro, al paradigma característico de la Web Social (contenidos personalizados y/o generados por el usuario, entornos abiertos a terceras partes, trabajo colaborativo, predominio de la lógica de la puesta en común, etc.). Ciertamente, puede hablarse, en un sentido amplio, del dispositivo móvil como una incipiente 'cuarta pantalla' de uso cotidiano (junto con las tres pantallas predominantes: cine, TV y PC-internet) (Ibid., 2008).

\section{"El móvil es una incipiente 'cuarta pantalla' de uso cotidiano después de las de cine, TV y PC-internet"}

La importancia creciente de los estándares de internet y el potencial de los dispositivos móviles en virtud de su conectividad ubicua, su capacidad de personalización y su omnipresencia confieren una especial importancia al modelo de la Web social en el desarrollo futuro, no ya sólo de contenidos y servicios, sino de la estructura del mercado mismo de datos en el entorno de los operadores y fabricantes (Jaokar; Fish, 2006; Berman et al., 2007; Iddris, 2007; Lane, 2008).

El presente artículo propone un marco general de los factores relevantes por los que el paradigma de la Web Social se perfila actualmente como un modelo prevalente en el desarrollo del mercado y el consumo de datos en dispositivos móviles, aportando una estructura conceptual de utilidad para el posterior estudio evolutivo de los modelos de producción, negocio y consumo de los contenidos móviles. Las propuestas recogidas en este texto forman parte de los resultados parciales del proyecto de investigación 'Movilsoc: Impacto social de la telefonía móvil en España, financiado por la Fundación Séneca y llevado a cabo por la Universidad de Murcia en colaboración con la University of New South Wales (Australia).

\section{Comunicación móvil e internet: precisiones terminológicas}

La profusión conceptual que a menudo acompaña las iniciativas tecnológicas exige algunas precisiones a la hora de usar los términos 'Internet' y 'Web' en relación al entorno móvil. Aunque originariamente 'Internet móvil' se utilizó para designar el acceso a internet a través del teléfono móvil (fundamentalmente con las conectividades WAP y GPRS que caracterizaron a la denominada $2.5 \mathrm{G}$ ), actualmente debe restringirse su uso al acceso a internet a través de conectividad 3G. En sentido estricto, por tanto, 'Internet móvil' designa la capacidad de acceder a internet a través de una conexión ubicua (proporcionada por los operadores 3G), por lo que tanto los dispositivos móviles como los PCs con conectividad 3G son operativos en el ámbito de la Internet móvil. 'Internet móvil', así, es algo distinto de 'Internet en el móvil'.

No obstante, la posibilidad misma del acceso a internet desde el dispositivo móvil hizo patente la necesidad de adaptar los estándares de la WWW a las características del dispositivo de bolsillo: menor tamaño y resolución de pantalla, menor capacidad de procesa- 
miento de datos, configuración vertical, problemas específicos de usabilidad en cuanto a entrada de datos y presentación de contenidos, etc. En más de un sentido los avances tecnológicos han permitido aproximar ambos mundos, no sólo simplificando la WWW, sino añadiendo complejidad a los propios dispositivos móviles: mayor capacidad multimedia, pantallas horizontales y táctiles que acercan el uso de la interfaz al del PC, mejora de la gestión de datos con navegadores basados en proxy (Pardo-Kuklinsky et al., 2008), mejora de la interfaz con el uso del zoom, etc. Al mismo tiempo, en los últimos siete años se ha producido una notable adaptación del entorno web a los dispositivos móviles, que dio sus primeros pasos con la tecnología WAP (básicamente adaptando la estructura de los portales web a navegación vertical basada en texto) y que, en la actualidad, se implementa en tres tendencias diferenciadas:

a) redireccionamiento hacia webs con formato móvil a partir de la identificación del dispositivo (smartphone, iPhone, PDA...);

b) identificación nominal del sitio web como específicamente diseñado para el móvil, generalmente a través de dominios (.mobi), subdominios (.m.com) o secciones de la url (/mobile); y

c) utilización de aplicaciones anidadas de conversión (como Joey o Mowser) que permiten generar una versión móvil ad hoc del sitio web.

Es, en definitiva, a este proceso de adaptación mutua entre los estándares de la WWW y el dispositivo móvil al que hace referencia el término 'Web móvil'. Prueba de la creciente importancia de la Web móvil es sin duda la proliferación de versiones móviles de los medios consolidados en la Web (desde Google y Yahoo, con sus múltiples aplicaciones, a la $B B C$ o la $C N N$ ). De acuerdo con el informe publicado por Opera Mini (2008), el crecimiento del acceso a la Web móvil en 2008 superó el 400\% (El país, 30/10/2008) y en países como Japón (con una estructura del mercado móvil característica), el acceso a la Web vía móvil es ya superior al acceso vía PC (Fitzpatrick, 2007).

Dadas las especiales características del dispositivo móvil -ubicuidad y vinculación con la identidad del usuario-, no puede extrañar que ese mismo proceso de adaptación entre la web y el entorno móvil confluya con especial énfasis en la denominada Web social. Las propias características del consumo de los contenidos móviles contribuyen a ello: el predominio de la lógica del 'compartir' - o la vinculación del disfrute de los contenidos con el hecho de compartirlos con otros-, la tendencia a la personalización, la adaptación del uso a la situación, etc. (Aguado; Martínez, 2008). La coincidencia en los perfiles del usuario de la Web 2.0 y del usuario intensivo del dispositivo móvil multimedia (los denominados 'nativos digitales') (Feijóo; Maghiros, 2008; Pardo Kuklinski et al., 2008) es también un factor a tener en cuenta, junto con la propia naturaleza de las innovaciones producidas o potenciadas por los usuarios en la comunicación móvil (SMS grupal, geocaching -uso del GPS como juego social de localización-, toothing -o utilización de la conectividad Bluetooth en redes sociales-, etc.). En 2009, 20 de los más de 100 millones de usuarios de Facebook acceden a la conocida red social a través de dispositivos móviles (Díaz, 2009), y fabricantes y operadores desarrollan aplicaciones para redes sociales (como Nokia con Vine y Mosh o Movistar con Keteke). La cuestión es si la Web 2.0 móvil se limita únicamente a la adaptación de las aplicaciones de la Web social al entorno móvil o si, por el contrario, asistimos a un nuevo proceso de doble transformación, de acuerdo con el cual, el entorno móvil introduce también cambios relevantes en la Web social.

\section{"La ubicuidad y vinculación con la identidad del usuario del entorno móvil introduce cambios relevantes en la Web social”}

\section{Dos paradigmas enfrentados}

La respuesta a esa cuestión pasa por reconocer que la confluencia entre el dispositivo móvil y la web social va más allá de una tendencia en los modelos de negocio o de una moda en los patrones de consumo. La coincidencia en los procesos de innovación, en los perfiles de usuario y en los valores percibidos por los usuarios respecto de las aplicaciones sociales y el dispositivo móvil (Aguado; Martínez, 2008; Feijóo y Maghiros, 2008; Ernest\&Young, 2007; Berman et al., 2006) apuntan más bien a una coincidencia de paradigmas, esto es, de maneras de entender la tecnología como mediación en la vida cotidiana.

En el proceso de mediatización del dispositivo móvil confluyen actualmente dos paradigmas contradictorios, en pugna por la definición de la naturaleza del nuevo medio (Aguado; Martínez, 2008), que responden, como mencionábamos más arriba, a la doble concepción del dispositivo móvil como plataforma de consumo cultural y como tecnología de relación (Castells et al., 2006):

\subsection{Lo mediático convencional}

De forma resumida, el paradigma mediático convencional concibe el dispositivo móvil como platafor- 
ma de acceso individual a contenidos estandarizados y se caracteriza, entre otros, por los siguientes rasgos:

a) Relevancia de la dimensión institucional: La existencia de una institución o marca dedicada a la producción y distribución de contenidos en el marco de los medios convencionales (televisión, prensa, cine, música...).

b) Concentración del control sobre la producción y la distribución, otorgando a las operadoras un papel de cuello de botella y generalizando el modelo de negocio del tipo 'coto cerrado' (o “jardín vallado”) (Jaokar; Fish, 2006; De Waele, 2006) que dificulta la innovación en software y en formatos de contenidos.

c) Centrado en el contenido: maximiza la relevancia de la distribución y el control del acceso y minimiza la importancia del usuario y el contexto de uso, las dos principales aportaciones de la Web 2.0 y del entorno móvil (Feijóo; Maghiros, 2008). El contenido es, además, un producto acabado y cerrado, sólo accesible para el consumo.

d) Demanda una legislación restrictiva como consecuencia de su dependencia de los modelos de distribución y de negocio de los medios convencionales (Feijóo; Maghiros, 2008; Aguado; Martínez, 2008).

e) Predominio de estándares tecnológicos cerrados/ propietarios: el modelo de "coto cerrado" se extiende a las plataformas y sistemas operativos, limitando el desarrollo de aplicaciones por terceras partes y la contribución del feed-back productivo de los propios usuarios (Jaokar; Fish, 2006; Pardo Kuklinski et al., 2008).

f) Diversidad de estándares y formatos: como consecuencia de (e), los diferentes actores generan una auténtica pléyade de estándares y formatos no compatibles que nuevamente tienden a lastrar la innovación aumentando los costes de producción de nuevo software y aplicaciones y fragmentando los nichos de mercado (Aguado; Martínez, 2008; Pardo Kuklinski et al., 2008).

g) Concepción pasiva del usuario, que se limita al consumo en situaciones y contextos predefinidos.

h) Operatividad basada en las funciones del dispositivo, que implica una disociación entre aplicaciones (gestión) y datos (contenido).

\subsection{El entorno 2.0}

Frente al mediático convencional, el paradigma 2.0 en relación con el móvil puede sintetizarse en los términos opuestos:

a) La web como plataforma (O'Reilly, 2007) permite reducir el impacto negativo de las limitaciones de memoria y procesamiento del móvil (Pardo Kuklinski et al., 2008). b) Arquitectura colaborativa (O'Reilly, 2007), independiente de la dimensión institucional, que favorece las alianzas entre actores diversos y la integración de los usuarios en los procesos de producción, potenciando la innovación y la capacidad de respuesta a la demanda de los usuarios.

c) Como consecuencia de (a), el control sobre la producción y la distribución se descentraliza y desjerarquiza entre desarrolladores y usuarios. Como ya ocurrió con internet, los operadores pierden en este contexto su posición privilegiada, quedando relegados al papel de suministradores de acceso a la plataforma.

d) Centrado en el usuario: el contenido no es el final de la cadena de valor. Ésta se enfoca a la experiencia del usuario.

e) Estándares tecnológicos abiertos (Jaokar; Fish, 2006), que favorecen la compatibilidad, la complementariedad y, especialmente, la polivalencia de las aplicaciones en distintos medios (PC, consola, móvil...).

\section{"La incidencia mutua del entorno móvil y la Web 2.0 ha sido bautizada como Móvil 2.0"}

f) Concepción activa del usuario: éste se convierte en productor de contenido y en desarrollador de aplicaciones. El contexto espacial (geolocalización) y social (situación) del usuario adquiere relevancia tanto en el diseño como en la difusión y uso de información (Jaokar; Fish, 2006).

g) Operatividad basada en el binomio contenido/ aplicación (Ibid.), independiente del dispositivo. El principio de la Web como plataforma (O'Reilly, 2007) adquiere carta de naturaleza en un entorno IP global que involucra medios diversos.

De este enfrentamiento entre los dos paradigmas depende que el futuro dispositivo móvil se parezca más a un reproductor multimedia de bolsillo o a un pequeño terminal de acceso a internet con capacidad multimedia. Aunque las expectativas de fabricantes y operadores apuntaron inicialmente hacia el primer modelo (siguiendo la estela de la Web 1.0 y de una TV móvil concebida como una televisión por cable miniaturizada), la propia evolución de la Web 2.0, las aplicaciones de software y la orientación del consumo de los usuarios con mayor potencial futuro (los nativos digitales) están reorientando el modelo hacia la segunda opción (Gaptel, 2006; Berman et al., 2006; Ernest\&Young, 2007). El resultado es, así, una incidencia mutua del entorno móvil y la Web 2.0 que algunos autores han bautizado como Móvil 2.0 (De Waele, 2006). 


\section{Móvil 2.0}

El término Mobile 2.0 fue usado por primera vez en 2006 en el marco de una serie de seminarios y conferencias organizados por Verizon Wireless y Orange dedicados a la adaptación de la Web 2.0 al entorno móvil. En su origen surge como una serie de iniciativas destinadas a aprovechar el nuevo potencial del acceso ubicuo a la Web enfocado hacia las redes sociales. Las condiciones socio-técnicas que marcan el arranque de esa originaria Web 2.0 móvil son la rápida penetración de la banda ancha móvil y de las redes inalámbricas (wifi), la creciente presencia en el mercado de dispositivos móviles duales (3G y wifi) y el establecimiento de tarifas planas de voz y datos que abaratan los costes de uso del dispositivo como terminal online.

La Web 2.0 móvil aparece, pues, como una iniciativa de adaptación y está marcada por el desembarco en el móvil de los grandes motores de la Web 2.0: Yahoo Mobile (con Yahoo Go!), Google (con Google Maps y Google News para móvil), YouTube, Flickr, MySpace, etc. Sin embargo, esa Web 2.0 móvil pronto empieza a ser algo más que una mera adaptación. En la medida en que se produce también una influencia de los rasgos del dispositivo móvil (ubicuidad, geolocalización, adaptación al perfil del usuario, adaptación al contexto) en los estándares de la Web 2.0, el término 'Mobile 2.0' redefine su sentido (De Waele, 2006): se trata ya no de la Web 2.0 adaptada al dispositivo móvil, sino de la movilidad incorporada al paradigma 2.0.

Si el motor de la Web 2.0 móvil fue su optimización por los operadores como forma de impulsar los servicios de banda ancha móvil, el verdadero desencadenante del Móvil 2.0 lo constituye la irrupción en 2007 de sistemas operativos y plataformas de software abiertos, que hacen posible que nuevos actores (incluidos los propios usuarios) integren en ellos aplicaciones de producción, integración, gestión y comunicación de contenidos en redes sociales móviles. Es el caso del sistema operativo Android (de Google), o las plataformas Yahoo Mobile y Widsets (de Nokia) para el diseño de widgets móviles. La propia Nokia crea en 2008 la Symbian Foundation con vistas a abrir sus sistema operativo al desarrollo de aplicaciones por terceras partes, algo que, de un modo mucho más controlado, ya había hecho Apple con su célebre iPhone.

A lo largo de 2007 (y en mayor medida a partir de que comienzan a generalizarse las tarifas planas de voz y datos por los operadores) han ido apareciendo nuevos servicios y aplicaciones móviles de microblogging (Jaiku, Twitter), llamadas de voz IP (Gizmo, Skype), localización (Loopt, Plazes, Google Latitude), mensajería (Funambol), información y reserva de entradas (Mobo), intercambio de archivos (Mystrands), gestión de medios (JuiceCaster), búsqueda de informa- ción (Plusmo), redes sociales (itsmy.com, Facebook), etc. Lo característico de estas propuestas es que contribuyen a redefinir el concepto de contenido móvil: ya no se trata sólo de datos sujetos a consumo pasivo, sino de datos en/con aplicaciones que integran funcionalidad y contenido (De Waele, 2006; Jaokar; Fish, 2007; Aguado; Martínez, 2008).

Uno de los factores decisivos del Móvil 2.0 es la capacidad de adaptación al lugar y la situación de uso a través de la geolocalización, pues abre el horizonte al intercambio de contenidos no sólo a partir de categorías predefinidas, sino de la situación física y el perfil del usuario (por ejemplo, abre la puerta a la utilización de etiquetas y mensajes vinculados a coordenadas geográficas). El concepto de 'sitio Web' se geolocaliza en el Móvil 2.0, de tal modo que uno puede colgar un post con su opinión sobre la comida de un restaurante o con recomendaciones sobre la visita a un museo no ya en su blog, sino en la coordenada geográfica del mismo restaurante o del museo, de modo que un usuario autorizado puede acceder a esa información en el contexto físico y la situación social en que fue creado. Técnicamente, pues, el Móvil 2.0 reubica la información en el punto de inspiración (Jaokar; Fish, 2006). La aplicación Vine de Nokia permite, por ejemplo, asociar información a rutas GPS, transformando el concepto mismo de 'mapa' en una suerte de diario de viaje físicamente situado, integrable a su vez en redes sociales como Mosh o Facebook.

\section{"La geolocalización permite transformar un 'mapa' en una suerte de diario de viaje físicamente situado"}

\section{Horizontes de desarrollo}

El proceso de transformación recíproca entre la Web 2.0 y el Móvil 2.0 implica, al menos, dos horizontes relevantes en relación a usuarios y operadores (dos actores tradicionalmente distantes en el mercado tradicional de las comunicaciones móviles):

Desde el punto de vista de los usuarios, la lógica en red de los contenidos sociales móviles se extiende a los hábitos de uso, favoreciendo rutinas y pautas de uso transversales a varios medios y/o tecnologías (integrando, por ejemplo, GPS, blogging, videostreaming $\mathrm{y}$ fotos en aplicaciones sociales operativas desde PC y desde móvil). En otros términos, la movilidad potencia notablemente la expansión de la Web como plataforma de mediación tecnológica. 
Desde el punto de vista de las operadoras, el Móvil 2.0 supone simultáneamente una oportunidad, una amenaza y un desafío. Una oportunidad, en cuanto incrementa exponencialmente el valor añadido de los servicios de conectividad (banda ancha móvil) y abre un horizonte de negocio (servicios de networking, alianzas con marcas de software social, etc.) en el que no sólo los operadores, sino también los fabricantes de dispositivos han puesto ya sus miras.

Una amenaza, por cuanto en el entorno 2.0 se dan modalidades de distribución, formatos y consumo alternativas a los contenidos tradicionales adaptados al móvil (Youtube móvil frente a la TV móvil, redes de contenidos frente a proveedores de música y juegos, agregadores de contenido frente a acceso restringido a medios proveedores de noticias, etc.). La fuerza de estas alternativas reside no sólo en la implicación de los usuarios, sino en su carácter gratuito y abierto y en su simplicidad, rasgos compartidos por la Web social y el entorno móvil. Por ejemplo, ¿para qué contratar el canal de la $B B C$ en un paquete de TV móvil si puedo acceder a los vídeos informativos de $B B C$ Mobile o $C N N$ Mobile a través de una aplicación que agrega vídeos informativos de diversos canales? Un informe reciente de Telecom.com (Newman, 2008) apunta precisamente en esta dirección, señalando que en el horizonte de 2013 los beneficios de las operadoras -en el ámbito del tráfico de datos y excluido el SMS- tendrán más que ver con los servicios básicos de acceso a internet que con los paquetes de acceso a contenidos adaptados.

Finalmente, el Móvil 2.0 supone un desafío para las operadoras por cuanto fuerza a abandonar $-\mathrm{o}$, al menos, a relativizar- el modelo 'coto cerrado' de negocio

\section{"Las operadoras tienen el reto de estar en plataformas abiertas que incentiven la participación de terceras partes"}

de contenidos (servicios de acceso restringido a contenidos puntuales) y plantea su participación en plataformas abiertas que incentivan la inclusión de terceras partes (desarrolladores de software social, agencias de publicidad móvil, etc.) y la innovación de formatos.

Finalmente, en la tabla 1 se sintetizan los rasgos característicos de los dos paradigmas esbozados en las páginas anteriores. Conviene notar que las diferencias implicadas en ellos suponen, a la postre, dos concepciones opuestas respecto del eje sobre el que se articula la convergencia de las comunicaciones móviles en el ecosistema mediático: de un lado, en el paradigma mediático, se trataría de una convergencia orientada desde y para los medios y formatos, mientras que desde el otro, desde el paradigma 2.0, se trataría de una convergencia orientada desde/para los usos. De ahí la relevancia que las estrategias de coordinación de medios diversos -crossmedia- (móvil, PC-internet, iPod, consolas, etc.) presentan en el caso del Móvil 2.0.

\section{Referencias}

Aguado, Juan Miguel; Martínez, Inmaculada. "The fourth screen and the liquid medium: notes for a characterization of the media cultures implicit in mobile entertainment contents". En: Hartmann, Maren; Rössler, Patrick. After the mobile phone? social change and the development of mobile communication. Berlín: Frank\&Timme, 2008, pp. 69-84, ISBN 978-3-86596167-9

\begin{tabular}{|c|c|}
\hline Paradigma mediático & Paradigma 2.0 \\
\hline Base institucional & La web como plataforma / estructura colaborativa \\
\hline Concentración del control sobre la producción y distribución & Control distribuido sobre la producción y distribución \\
\hline El contenido es el centro & El usuario es el centro \\
\hline Demanda fuerte legislación específica & Suficiencia de los marcos legales estándar \\
\hline Estándares tecnológicos cerrados / propietarios & Estándares tecnológicos abiertos \\
\hline Ausencia de innovación en estándares y hábitos de uso & Innovación en formatos y hábitos de uso \\
\hline Concepción pasiva del usuario & Concepción activa del usuario \\
\hline Operatividad basada en las funciones del dispositivo & $\begin{array}{l}\text { Operatividad basada en el binomio [contenidos }+ \\
\text { aplicaciones] que ponen en juego la coordinación de } \\
\text { medios diversos (crossmedia) }\end{array}$ \\
\hline \multicolumn{2}{|l|}{ Concepción de la comunicación móvil } \\
\hline Plataforma de consumo de contenidos cerrados & Plataforma de comunicación \\
\hline \multicolumn{2}{|l|}{ Tipo de convergencia implicada } \\
\hline Convergencia de medios / formatos & Convergencia de usos \\
\hline
\end{tabular}

Tabla 1: Conceptos opuestos de los paradigmas mediático y 2.0 en la implementación de las comunicaciones móviles 
Aguado, Juan Miguel; Martínez, Inmaculada. "From mobile phones to mobile media: current developments in mobile phone-based cultural consumption". En: Goggin, Gerard; Hjorth, Larissa. Mobile media 2007. Sydney: University of New South Wales, 2007, pp. 47-57, ISBN 9781864878905

Aguado, Juan Miguel; Martínez, Inmaculada. "La mediatización de la telefonía móvil: de la interacción al consumo cultural". ZER, Revista de estudios de comunicación, 2006, mayo, v. 11, n. 20, pp. 319-343.

Berman, Saul J.; Steinberg, Adam R.; Shipnuck, Louisa A. The end of television as we know it: a future industry perspective. IBM Institute for Business Value. 2006.

http://www-05.ibm.com/e-business/uk/innovation/channel/html/revitalize/ trends/pdf/ge510-6248-end-of-tv-full.pdf

Berman, Saul J.; Battino, William; Shipnuck, Louisa A.; Neus, Andreas The end of advertising as we know it. IBM Institute for Business Value 2007.

http://www-03.ibm.com/industries/media/doc/content/bin/media_ibv_ advertisingv2.pdf

Castells, Manuel; Fernández-Ardévol, Mireia; Linchuan Qiu, Jack; Sey, Araba. Comunicación móvil y sociedad. Una perspectiva global. Barcelona: Ariel, 2006, ISBN: 8434453274

CMT. Informe anual 2007. Comisión del Mercado de las Telecomunicaciones.

http://www.cmt.es/es/publicaciones/anexos/Informe_anual_CMT_2007_ web.pdf

De Waele, Rudy. Understanding Mobile 2.0. Publicado en 2006. http://www.readwriteweb.com/archives/understanding_mobile_2.php

Ernest\&Young. Mediabarometer 2007.

http://www.ey.nl/?pag=956\& nieuws_id $=3058$

Díaz, Bruno. "20 millones de usuarios acceden a Facebook vía móviles". Wayerless, 10/01/2009.

http://www.wayerless.com/2009/01/20-millones-de-usuarios-acceden-afacebook-via-moviles/

Feijóo, Claudio; Maghiros, Ioannes. Mobile content. On the verge of an explosion. JRC scientific reports, EC. Sevilla, Institute for Prospective Technological Studies, Nov. 2008.

Feldmann, Valerie. Leveraging mobile media. Cross-media strategy and innovation policy for mobile media communication. Heidelberg-New Cork: Physica Verlag, 2005, ISBN 978-3-7908-1575-7

Fitzpatrick, Michael. "Why mobile Japan leads the World". The guardian, 27/12/2007.

http://www.guardian.co.uk/technology/2007/sep/27/guardianweeklytechno logysection.mobilephones
Fortunati, Leopoldina; Pozzobon, Fabio. "Media mobiles: when interpersonal media become mass media". ICA pre-conference "After the mobile phone?", 2006, University of Erfurt, pp. 205-219.

Gaptel (Grupo de Análisis y Prospectiva del Sector de las Telecomunicaciones). Contenidos digitales: nuevos modelos de distribución online, 2006.

http://observatorio.red.es/estudios/documentos/ContenidosDigitales_final. $p d f$

Iddris, Faisal. Mobile advertising in B2C marketing. Tesis doctoral. Lulea University of Technology, 2006.

http://epubl.luth.se/1653-0187/2006/11/LTU-PB-EX-0611-SE.pdf

Jaokar, Agit; Fish, Anthony. Mobile Web 2.0. Oxford: Futuretext Publications, 2006, ISBN 13: 978-0954432768

Katz, James; Satomi, Steven. "Mobile phones as fashion statements: the co-creation of mobile communication's public meaning". En: Ling, Rich; Pedersen, Per. Mobile communications: re-negotiation of the social sphere. Surrey, UK: Springer, 2005, pp. 63-81.

Lane, Nathan. Mobile advertising: cutting through the hype. London, Informa Media \& Telecom, May 2008, 226 pp.

Olswang. Olswang convergence consumer survey 2007. http://www.olswang.com/convergence07/convergence2007.zip

Opera Mini. State of the mobile web report in 2008. http://www.opera.com/smw/

O'Reilly, T. (2007): "What is the Web 2.0? Design patterns and business models for the next generation of software". Communications and strategies, 2007, n. 1, pp. 17-37.

Pardo-Kuklinski, Hugo; Brandt, Jack; Puerta, Juan-Pablo. "Mobile Web 2.0: a theoretical-technical framework and developing trends". International journal of interactive mobile technologies, 2008, v. 2, n. 4, pp. 54-61.

Telefónica. Informe sobre la sociedad de la información en España 2008 (SIE-2008). Madrid, Ariel/Fundación Telefónica, 2008

http://www.telefonica.es/sociedaddelainformacion/html/informes_home. shtml\#

Juan-Miguel Aguado-Terrón e Inmaculada J. Martínez-Martínez, Facultad de Comunicación y Documentación, Universidad de Murcia

Campus de Espinardo, s/n. 30100 Murcia.

jmaguado@um.es

inmartin@um.es

\section{Suscripciones}

Renovar (o comenzar) la suscripción a "El profesional de la información" es ágil y sencillo.

Usted puede gestionar online su suscripción conectándose a esta web: http://www.elprofesionaldelainformacion.com/suscripciones.html

Si lo desea puede comunicar con nosotros dirigiéndose a: suscripciones@elprofesionaldelainformacion.com

o al teléfono: +34-609352954 